\title{
Efeito da Idade Materna sobre os Resultados Perinatais
}

\author{
Effect of Maternal Age on Perinatal Outcomes
}

\begin{abstract}
George Dantas de Azevedo, Reginaldo Antonio de Oliveira Freitas Júnior, Ana Karla Monteiro Santana de Oliveira Freitas, Ana Cristina Pinheiro Fernandes de Araújo, Elvira Maria Mafaldo Soares, Técia Maria de Oliveira Maranhão
\end{abstract}

\section{RESUMO}

Objetivo: analisar a relação entre a idade materna e a ocorrência de resultados perinatais adversos na população do Rio Grande do Norte.

Métodos: foram analisados os registros oficiais de 57.088 nascidos vivos no Estado do Rio Grande do Norte no ano de 1997. Os dados foram obtidos do Sistema de Informação sobre Nascidos Vivos do Ministério da Saúde. A população estudada foi dividida em Grupos I, II e III, segundo a faixa etária materna: 10 a 19, 20 a 34 e 35 anos ou mais, respectivamente. As variáveis analisadas foram: duração da gestação, peso ao nascer e tipo de parto. A análise estatística foi realizada utilizando-se o teste $\chi^{2}$.

Resultados: observamos uma maior incidência de parto pré-termo no Grupo I (4,3\%), em comparação ao Grupo II $(3,7 \%)(p=0,0028)$. A taxa de cesariana foi menor nos Grupos I e III, em comparação ao Grupo II ( $p<0,0001)$. Evidenciamos freqüência significativamente maior de recém-nascidos de baixo peso nos Grupos I (8,4\%) e III (8,3\%), quando comparados ao Grupo II $(6,5 \%)(p<0,0001)$.

Conclusões: a gravidez nos extremos da vida reprodutiva esteve associada com maior freqüência de parto pré-termo e baixo peso ao nascer, entretanto, com relação ao tipo de parto, foi observada maior freqüencia de parto normal do que no grupo de gestantes com idade entre 20 e 34 anos.

PALAVRAS-CHAVE: Idade materna. Resultados perinatais. Adolescência. Gravidez normal. Cesárea.

\section{Introdução}

Relatos de estudos prévios sobre a relação entre a idade materna e os resultados perinatais são extremamente controversos. No grupo de gestantes adolescentes, vários autores têm relatado maior incidência de complicações obstétricas e perinatais, tais como baixo peso ao nascer, parto pré-termo, amniorrexe prematura, pré-eclâmpsia e diabetes gestacional ${ }^{1-4}$. Estudos mais recentes, entretanto, têm sugerido que os resultados adversos descritos nesta população traduzem mais claramente as condições sociais e de saúde às quais acha-se submetida, que propriamente o

Universidade Federal do Rio Grande do Norte Correspondência:

Técia Maria de Oliveira Maranhão

Av. Deodoro, 240/102 - Petrópolis

59012-600 - Natal - RN status adolescente s-9. $^{\text {. }}$

Atualmente, considera-se que primigestas adolescentes, com idade entre 16 e 19 anos e que recebem cuidados pré-natais adequados, não apresentam risco perinatal aumentado, quando comparadas a mulheres mais velhas em condições sociais e econômicas similares. Por outro lado, este quadro aparentemente favorável do ponto de vista obstétrico não anula as significativas conseqüências psicológicas e sócio-econômicas que uma gravidez na adolescência pode acarretar. Conforme destacado por Card $\&$ Wise $^{10}$, as mulheres que tiveram filhos durante a adolescência estão submetidas a um risco aumentado de descontinuacão dos estudos, divórcio, gravidezes repetidas e maior chance de pobreza.

Em relação à gravidez em mulheres com idade superior aos 35 anos, existe um conceito geral acerca de um maior risco obstétrico. Isto seria 
decorrente tanto da própria senescência ovariana quanto da freqüência aumentada de doenças crônicas em mulheres nessa faixa etária, notadamente a hipertensão arterial e diabetes mellitus, que sabidamente acarretam riscos potenciais para a gravidez. Particularmente no grupo de gestantes com idade superior a 45 anos, a literatura demonstra maior freqüência de complicações pré-gestacionais (hipertensão crônica e hipotireoidismo) e gestacionais (anormalidades genéticas, diabetes gestacional, maior incidência de cesariana e macrossomia fetal), a despeito de bons resultados obstétricos e perinatais ${ }^{11}$.

O propósito deste estudo foi investigar a relação entre idade materna precoce ou avançada e os resultados perinatais na população de mulheres grávidas do Rio Grande do Norte.

\section{Pacientes e Métodos}

Foram analisados os dados oficiais obtidos do sistema computadorizado de informações de saúde do Ministério da Saúde do Brasil (DATASUS), referentes ao Sistema de Informações sobre Nascidos Vivos (SINASC), relativos ao Estado do Rio Grande do Norte, ano de 1997, totalizando 57.088 registros de nascidos vivos (NV). Os dados obtidos foram analisados visando à obtenção dos cruzamentos estatísticos entre as variáveis consideradas e, para fins de análise estatística foram considerados apenas os dados adequadamente registrados para cada variável, sendo desprezadas informações classificadas como 'ignorado', 'inexistente' ou 'outros'.

Os grupos de estudo foram estratificados, de acordo com a idade materna, em três categorias: o Grupo I englobou as gestantes adolescentes (faixa etária dos 10 aos 19 anos), o Grupo II (Grupo controle) compreendeu as mulheres na faixa etária entre 20 e 34 anos e o Grupo III foi constituído de mulheres com gravidez em idade avançada (35 anos ou mais).

Foram analisados a duração da gestação, peso do recém-nascido ao nascimento e tipo de parto, estratificados qualitativamente conforme a padronização na declaração de NV. Para a variável "duração da gestação" foram consideradas as seguintes possibilidades: o parto ocorrido antes de 37 semanas completas de gravidez foi classificado como pré-termo, de 37 a 42 semanas completas como parto a termo e a gravidez prolongada foi definida como aquela cujo parto ocorreu após 42 semanas de gestação. Quanto a variável "peso ao nascimento", o peso adequado foi considerado como aquele situado na faixa entre 2.500 e $3.999 \mathrm{~g}$, atri- buindo-se as expressões "baixo peso" aos recémnascidos com peso inferior a $2.500 \mathrm{~g} \mathrm{e}$ "sobrepeso" aqueles com peso igual ou superior a $4.000 \mathrm{~g}$. Com relação ao "tipo de parto", foram analisadas as freqüências de parto normal, cesárea e fórcipe.

As freqüências de cada variável nos grupos de mulheres adolescentes (Grupo I) e de idade avançada (Grupo III) foram comparadas com as respectivas freqüências no grupo controle constituído de mulheres com idade entre 20 e 34 anos (Grupo II). Para a análise estatística foi utilizado o teste $\chi^{2}$, visando a determinar a possivel significância das diferenças entre os Grupos I e III em relação ao Grupo II, sendo adotado um nível de significância de $5 \%$. As análises foram realizadas utilizando o "software" Graphpad Prism 3.0.

\section{Resultados}

A Figura 1 apresenta a distribuição dos NV de acordo com a idade materna, onde se observa que $25,3 \%$ dos partos ocorreram em adolescentes, $66,5 \%$ em mulheres pertencentes ao Grupo II e $8,2 \%$ em mulheres com idade maior ou igual a 35 anos.

(\%)
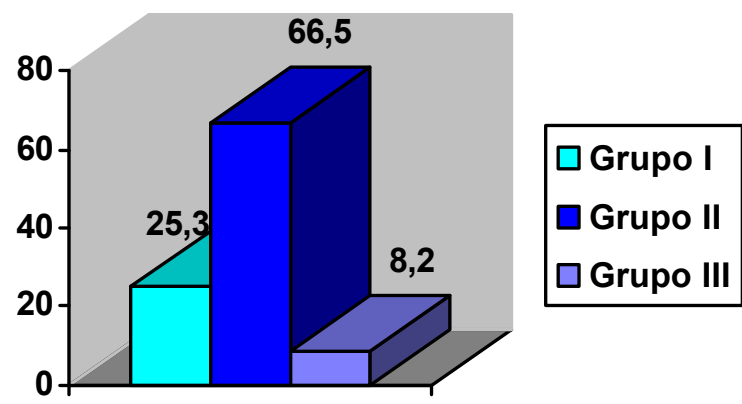

Figura 1 - Distribuição do total de nascidos vivos segundo a faixa etária materna. Rio Grande do Norte, 1997.

Em relação ao tipo de parto, foi evidenciada uma incidência de parto normal significativamente maior nos Grupos I $(78,2 \%)$ e III $(72,6 \%)$, quando comparados ao Grupo II $(70,1 \%)$ $(\mathrm{p}<0,0001)$. Analisando-se o percentual de cesárea entre os grupos, conforme ilustrado na Tabela 1, observou-se uma menor freqüência no grupo das adolescentes $(20,3 \%)$ em relação aos Grupos II e III (29,0 e $27,0 \%$, respectivamente), o que também foi significativo do ponto de vista estatístico. Foi evidenciada, ainda, uma incidência discretamente maior de parto fórcipe no Grupo I, em relação aos outros grupos. 
Tabela 1 - Distribuição dos nascidos vivos segundo o tipo de parto e a faixa etária materna no Estado do Rio Grande do Norte (1997). Evidenciam-se maiores taxas de parto normal e menores taxas de cesariana nos Grupos I e III, em comparação ao grupo controle.

\begin{tabular}{lrrrrrrrr}
\hline Tipo de parto & \multicolumn{2}{c}{ Grupo I } & \multicolumn{2}{c}{ Grupo II } & \multicolumn{2}{c}{ Grupo III } & \multicolumn{2}{c}{ Total } \\
& \multicolumn{1}{c}{} & \multicolumn{1}{c}{$\%$} & \multicolumn{1}{c}{$\mathbf{n}$} & \multicolumn{1}{c}{$\%$} & $\mathbf{n}$ & \multicolumn{1}{c}{$\%$} & \multicolumn{1}{c}{$\mathbf{n}$} & \multicolumn{1}{c}{$\%$} \\
\hline Normal & 10.993 & $78,2^{*}$ & 26.005 & 70,1 & 3.312 & $72,6^{* *}$ & 40.310 & 72,3 \\
Cesárea & 2852 & $20,3^{*}$ & 10.751 & 29,0 & 1.232 & $27,0^{* *}$ & 14.835 & 26,6 \\
Fórcipe & 217 & 1,5 & 335 & 0,9 & 17 & 0,4 & 569 & 1,1 \\
Total & 14.061 & 100,0 & 37.091 & 100,0 & 4.561 & 100,0 & 55.714 & 100,0 \\
\hline
\end{tabular}

${ }^{*} p<0,0001$ (Grupo I x Grupo II)

** $p<0,0001$ (Grupo III x Grupo II)

Fonte: DATASUS/SINASC/Ministério da Saúde - Brasil.

Grupo I: 10-19 anos; Grupo: II 20-34 anos e Grupo III: mais de 35 anos

A Tabela 2 apresenta a distribuição dos nascimentos de acordo com a duração da gestação. Observa-se a ocorrência de parto pré-termo em $4,3 \%$ das gestantes adolescentes, uma freqüência significativamente maior do que a observada no Grupo II $(3,7 \%)(p=0,0028)$. Em relação à gravidez prolongada, nenhuma diferença estatisticamente significativa foi evidenciada entre os grupos.

Tabela 2 - Distribuição dos nascidos vivos de acordo com a duração da gestação e a faixa etária materna no Estado do Rio Grande do Norte (1997). Observa-se freqüência de partos pré-termo significativamente mais elevada no grupo de gestantes adolescentes, em comparação ao grupo controle.

\begin{tabular}{lrrrrrrrr}
\hline Duração da & \multicolumn{2}{c}{ Grupo I } & \multicolumn{2}{c}{ Grupo II } & \multicolumn{2}{c}{ Grupo III } & \multicolumn{2}{c}{ Total } \\
estação (semanas) & \multicolumn{1}{c}{$\mathbf{n}$} & \multicolumn{1}{c}{$\%$} & \multicolumn{1}{c}{$\mathbf{n}$} & \multicolumn{1}{c}{$\%$} & \multicolumn{1}{c}{$\mathbf{n}$} & \multicolumn{1}{c}{$\%$} & \multicolumn{1}{c}{$\mathbf{n}$} & \multicolumn{1}{c}{$\%$} \\
\hline Até 36 & 612 & $4,3^{*}$ & 1.378 & 3,7 & 190 & 4,2 & 2.180 & 3,9 \\
37-41 & 13.176 & 94,3 & 34.942 & 94,8 & 4.274 & 94,4 & 52.392 & 94,6 \\
42 e mais & 190 & 1,4 & 541 & 1,5 & 63 & 1,4 & 794 & 1,5 \\
Total & 13.978 & 100,0 & 36.861 & 100,0 & 4.527 & 100,0 & 55.366 & 100,0 \\
\hline
\end{tabular}

${ }^{*} p=0,0028$ (Grupo I x Grupo II)

Fonte: DATASUS/SINASC/Ministério da Saúde - Brasil.

Grupo I: 10-19 anos; Grupo II: 20-34 anos e Grupo III: mais de 35 anos

Procedendo-se à análise com relação ao peso dos recém-nascidos, detectou-se uma maior incidência de baixo peso ao nascimento nos Grupos I $(8,4 \%)$ e III $(8,3 \%)$, quando comparados ao Grupo II
(6,5 \%). Estas comparações apresentaram relevância estatística, com $\mathrm{p}<0,0001$. A freqüência de recém-nascidos com peso superior a $4.000 \mathrm{~g}$ foi maior no Grupo III (10,7\%) (Tabela 3).

Tabela 3 - Distribuição dos nascidos vivos de acordo com o peso ao nascimento e a faixa etária materna no Estado do Rio Grande do Norte, 1997. Observam-se maiores freqüências de baixo peso ao nascimento nos Grupos I e III, e de recém-nascidos com peso acima de $4.000 \mathrm{~g}$ no Grupo III.

\begin{tabular}{lrrrrrrrr}
\hline Peso ao nascimento & \multicolumn{2}{c}{ Grupo I } & \multicolumn{2}{c}{ Grupo II } & \multicolumn{2}{c}{ Grupo III } & \multicolumn{2}{c}{ Total } \\
(gramas) & \multicolumn{1}{c}{$\mathbf{n}$} & \multicolumn{1}{c}{$\%$} & \multicolumn{1}{c}{$\mathbf{n}$} & \multicolumn{1}{c}{$\%$} & \multicolumn{1}{c}{$\mathbf{n}$} & \multicolumn{1}{c}{$\%$} & \multicolumn{1}{c}{$\mathbf{n}$} & $\%$ \\
\hline$<2.500 \mathrm{~g}$ & 1.175 & $8,4^{*}$ & 2.407 & 6,5 & 373 & $8,3^{* *}$ & 3.955 & 7,1 \\
$2.500-3.999 \mathrm{~g}$ & 12.093 & 86,5 & 31.313 & 84,9 & 3.641 & 81,0 & 47.047 & 85,0 \\
4.000 g e mais & 716 & $5,1^{*}$ & 3.162 & 8,6 & 482 & $10,7^{* *}$ & 4.360 & 7,9 \\
Total & 13.984 & 100,0 & 36.882 & 100,0 & 4.496 & 100,0 & 55.362 & 100,0 \\
\hline
\end{tabular}

${ }^{*} p<0,0001$ (Grupo I x Grupo II)

${ }^{* *} p<0,0001$ (Grupo III x Grupo II)

Fonte: DATASUS/SINASC/Ministério da Saúde - Brasil.

Grupo I: 10-19 anos; Grupo II: 20-34 anos e Grupo III: mais de 35 anos

\section{Discussão}

Este estudo teve como objetivo principal comparar os resultados perinatais entre gestantes nas diversas faixas etárias, procurando investigar a maior freqüência de resultados adversos nas mulheres com gravidez nos extremos da vida reprodutiva. Apesar dos inúmeros relatos existentes na literatura, atribuindo um maior risco a es- 
sas mulheres, o assunto ainda permanece bastante controverso. Na atualidade, vem sendo destacado que mais importante do que a idade, seriam as condições de vida e saúde das gestantes, principalmente, a qualidade da assistência obstétrica no pré-natal e no parto ${ }^{12}$.

Diversos autores apontam para um risco aumentado de parto operatório ${ }^{13-16}$ no grupo de gestantes adolescentes. Isto poderia ser explicado pela maior incidência de complicações neste grupo particular de mulheres, especialmente a prematuridade, baixo peso ao nascimento e retardo de crescimento intra-uterino, que, freqüentemente, constituem indicações para resolução da gravidez por via abdominal. Assim, a maior freqüência de parto operatório entre as adolescentes dependeria muito mais das complicações citadas do que da imaturidade física das jovens mães.

Neste estudo, a incidência de cesariana foi significativamente menor nas adolescentes, quando comparadas ao Grupo II. Esse resultado está em concordância com relatos recentes, nos quais se observaram maiores taxas de parto espontâneo e menores incidências de cesariana nas adolescentes $^{17,18}$. Lao e Ho ${ }^{19}$, comparando os resultados obstétricos de primíparas adolescentes com aqueles de mulheres na faixa etária dos 20 aos 34 anos, em que para cada caso era considerado um controle atendido no mesmo dia e pela mesma equipe médica, confirmaram os resultados acima destacados, evidenciando maior taxa de parto normal e menor de cesariana no grupo de adolescentes, mesmo naquelas com idade inferior a 16 anos.

Nas gestantes com idade de 35 anos ou mais, também têm sido descritas taxas mais altas de partos operatórios ${ }^{11,20,21}$. No presente trabalho, à semelhança das adolescentes, observamos maior incidência de parto normal e menor percentual de cesariana nas mulheres com gravidez em idade avançada. Em estudo semelhante, Milner et al. ${ }^{22}$ também não evidenciaram aumento das porcentagens de cesariana no grupo de gestantes com idade superior a 40 anos, a despeito da maior freqüência de complicações nesse grupo, como diabetes gestacional, hemorragia anteparto, sofrimento fetal e anomalias congênitas.

A maior ocorrência de parto pré-termo no grupo de adolescentes $(4,3 \%)$, significativamente relevante em relação ao grupo II, está em concordância com inúmeros relatos da literatura ${ }^{1-4}$. Entretanto, alguns estudos mostram incidências bem maiores do que a observada, conforme destacam Bozkaya et al. ${ }^{23}(9,3 \%)$ e Lao e $\mathrm{Ho}^{17}(13,0 \%)$. Propõe-se a hipótese de que o parto pré-termo nas adolescentes poderia ser considerado uma forma de resposta adaptativa à imaturidade fisica dessas mulheres, visando assegurar melhor prognós- tico a fetos menores, uma vez que nenhuma diferença significativa na morbimortalidade perinatal foi demonstrada ${ }^{17}$.

Embora tenha sido freqüentemente relatado que o baixo peso ao nascimento seja mais freqüente entre os recém-nascidos de mães adolescentes $^{1-4,17,23}$, pouco tem sido investigado sobre o quanto isso se deve à prematuridade ou redução do crescimento intra-uterino. Neste estudo, evidenciou-se um percentual de $8,4 \%$ de recém-nascidos de baixo peso no grupo de adolescentes, o que foi significativamente superior ao observado no Grupo II $(6,5 \%)$. Outro achado importante do presente estudo, corroborando o relato previamente publicado por Bozkaya et al. ${ }^{23}$, foi a freqüência de baixo peso ao nascimento maior do que a freqüência de partos pré-termo, uma situação que poderia ser atribuída ao baixo nível socioeconômico e deficiente aporte nutricional na população estudada.

Em estudo realizado por Elster ${ }^{24}$, demonstrou-se que o risco de restrição de crescimento intra-uterino é aumentado para adolescentes muito jovens (menores de 15 anos) e para aquelas que receberam cuidados pré-natais tardios. Entretanto, esse autor ressalta que a paridade exerce influência importante sobre a determinação do risco, o qual aumenta conforme o número de gestações prévias, independente da época de início do pré-natal.

No grupo de mulheres com idade maior que 35 anos, foram mais freqüentes tanto os recémnascidos de baixo peso ao nascimento, quanto os recém-nascidos com peso superior a $4.000 \mathrm{~g}$, confirmando os resultados da literatura ${ }^{21,22}$.

Os dados apresentados reconhecem a gestação nos extremos da vida reprodutiva como associada a uma maior freqüência de resultados perinatais adversos, notadamente o parto pré-termo e o baixo peso ao nascimento. A despeito dos resultados perinatais observados, a idade materna não se mostrou associada a piores resultados obstétricos, tendo em vista os maiores percentuais de partos normais observados entre as adolescentes e naquelas com idade superior a 35 anos.

\section{ABSTRACT}

Purpose: to investigate the interactions between maternal age and adverse perinatal outcomes in the State of Rio Grande do Norte.

Methods: we analyzed official records of 57,088 infants in the State of Rio Grande do Norte, from January 1997 to December 1997. Data were obtained from the Information 
System of the Health Ministry, Brazil. The sample was divided into three Groups I, II and III according to maternal age range: 10 to 19 years, 20 to 34, and 35 or more, respectively. The main outcome variables were: length of pregnancy, birth weight and mode of delivery. Statistical analysis was performed using $\chi^{2}$ test.

Results: preterm deliveries were $4.3 \%$ in the adolescent group vs $3.7 \%$ in Group II ( $p=0.0028)$. The incidence of cesarean section was higher in Group II than in the other Groups $(p<0.001)$. Low birth weight was significantly higher in Groups I (8.4\%) and III (8.3\%) when compared with Group II $(6.5 \%)(p<0.0001)$.

Conclusions: we found a higher incidence of lower birth weight and preterm delivery in the adolescent group. In women $\geq 35$ years old there was a high incidence of low birth weight and macrosomia. Results suggest that cesarean sections are more common in women aged 20-34 years than in adolescent and older mothers.

KEY WORDS: Maternal age. Perinatal outcome. Adolescent pregnancy. Cesarean section.

\section{Referências}

1. Coates JB $3^{\text {rd }}$. Obstetrics in the very young adolescent. Am J Obstet Gynecol 1970; 108:68-72.

2. Duenhoelter JK, Jimenez JM, Baumann G. Pregnancy performance of patients under fifteen years of age. Obstet Gynecol 1975; 46:49-52.

3. Goldberg GL, Craig CJ. Obstetric complications in adolescent pregnancies. S Afr Med J 1983; 64:863-4.

4. Leppert PC, Namerow PB, Barker D. Pregnancy outcomes among adolescent and older women receiving comprehensive prenatal care. J Adolesc Health Care1986; 7:112-7.

5. Poma PA. Effect of maternal age on pregnancy outcome. J Natl Med Assoc 1981; 73:1031-8.

6. Zuckerman B, Alpert JJ, Dooling E, et al. Neonatal outcome: is adolescent pregnancy a risk factor? Pediatrics 1983; 71:489-93.

7. Lubarsky SL, Schiff E, Friedman AS, Mercer BM, Sibai BM. Obstetric characteristics among nulliparas under age 15. Obstet Gynecol 1994; 84:365-8.

8. Satin AJ, Leveno KJ, Sherman ML, Reedy NJ, Lowe TW, McIntire DD. Maternal youth and pregnancy outcomes: middle school versus high school age groups compared with women beyond the teen years. Am J Obstet Gynecol 1994; 171:184-7.

9. Berenson AB, Wiemann CM, McCombs SL. Adverse perinatal outcomes in young adolescents. J Reprod Med 1997; 42:559-64.
10. Card JJ, Wise LL. Teenage mothers and teenage fathers: the impact of early childbearing on the parents' personal and professional lives. Fam Plann Perspect 1978; 10:199-205.

11.Dildy GA, Jackson GM, Fowers GK, Oshiro BT, Varner MW, Clark SL. Very advanced maternal age: pregnancy after age 45. Am J Obstet Gynecol 1996; 175:668-74.

12.Bukulmez O, Deren O. Perinatal outcome in adolescent pregnancies: a case-control study from a Turkish university hospital. Eur J Obstet Gynecol Reprod Biol 2000; 88:207-12.

13.Bacci A, Manhica GM, Machungo F, Bugalho A, Cuttini M. Outcome of teenage pregnancy in Maputo, Mozambique. Int J Gynaecol Obstet 1993; 40:19-23.

14.Khwaja SS, Al-Sibai MH, Al-Suleiman AS, el-Zibdeh MY. Obstetric implications of pregnancy in adolescence. Acta Obstet Gynecol Scand 1986; 65:57-61.

15.Konje JC, Palmer A, Watson A, Hay DM, Imrie A, Ewings P. Early teenage pregnancies in Hull. Br J Obstet Gynaecol 1992; 99:969-73.

16. Moerman ML. Growth of the birth canal in teenage girls. Am J Obstet Gynecol 1982; 143:528-32.

17.Lao TT, Ho LF. The obstetric implications of teenage pregnancy. Hum Reprod 1997; 12:2303-5.

18.Perry RL, Mannino B, Hediger ML, Scholl TO. Pregnancy in early adolescence: are there obstetric risks? J Matern Fetal Med 1996; 5:333-9.

19.Lao TT, Ho LF. Obstetric outcome of teenage pregnancies. Hum Reprod 1998; 13:3228-32.

20.Ales KL, Druzin ML, Santini DL. Impact of advanced maternal age on the outcome of pregnancy. Surg Gynecol Obstet 1990; 171:209-16.

21.Spellacy WN, Miller SJ, Winegar A. Pregnancy after 40 years of age. Obstet Gynecol 1986; 68:452-4.

22.Milner M, Barry-Kinsella C, Unwin A, Harrison RF. The impact of maternal age on pregnancy and its outcome. Int J Gynaecol Obstet 1992; 38:281-6.

23.Bozkaya H, Mocan $H$, Usluca $H$, Beser $E$, Gumustekin D. A retrospective analysis of adolescent pregnancies. Gynecol Obstet Invest 1996; 42:146-50.

24.Elster AB. The effect of maternal age, parity, and prenatal care on perinatal outcome in adolescent mothers. Am J Obstet Gynecol 1984; 149:845-7. 